\title{
On Grünbaum's Conjecture about Inner Illumination of Convex Bodies*
}

\author{
V. Boltyanski, ${ }^{1}$ H. Martini, ${ }^{2}$ and V. Soltan ${ }^{3}$ \\ ${ }^{1}$ El Centro de Investigacion en Matematicas, \\ A.P. 402,36000 Guanajuato, GTO, Mexico \\ ${ }^{2}$ Fakultät für Mathematik, TU Chemnitz, \\ D-09107 Chemnitz, Germany \\ ${ }^{3}$ Department of Mathematical Sciences, \\ George Mason University, \\ 4400 University Drive, \\ Fairfax, VA 22030, USA
}

\begin{abstract}
In 1964 Grünbaum conjectured that any primitive set illuminating from within a convex body in $E^{d}, d \geq 3$, has at most $2^{d}$ points. This was confirmed by V. Soltan in 1995 for the case $d=3$. Here we give a negative answer to Grünbaum's conjecture for all $d \geq 4$, by constructing a convex body $K \subset E^{d}$ with primitive illuminating sets of an arbitrarily large cardinality.
\end{abstract}

\section{Introduction and Main Results}

In what follows, $K$ means a convex body (i.e., a compact convex set with nonempty interior) in the $d$-dimensional linear space $E^{d}$. By bd $K$ and int $K$ we denote the boundary and the interior of $K$, respectively; rint $F$ means the relative interior of a convex set $F \subset E^{d}$ and conv $G$ denotes the convex hull of a set $G \subset E^{d} ;[x, z]$ and $] x, z[$ stand for the closed and open line segments with the endpoints $x$ and $z$, and $\langle x, z\rangle$ denotes the scalar product of $x$ and $z$.

The concept of illumination of a convex body from within (inner illumination, in other terminology) was introduced by P. Soltan [8]. A set $F \subset$ bd $K$ illuminates a convex body $K$ from within provided for every point $x \in$ bd $K$ there is a point $y \in F$ distinct from $x$ such that the open line segment ] $x, y$ [ is contained in int $K$. Soltan [8] proved that any

\footnotetext{
* V. Boltyanski and V. Soltan were supported by Deutsche Forschungsgemeinschaft.
} 
convex body $K$ in $E^{d}, d \geq 1$, can be illuminated from within by at most $d+1$ points, with exactly $d+1$ points if and only if $K$ is a simplex. (See the monograph [1] for a wider discussion on inner and exterior illumination of convex bodies.)

Following Grünbaum [3], a set $F$ illuminating a convex body $K$ from within is called primitive if no proper subset of $F$ illuminates $K$ from within. For short, we will say that $F$ is a primitive illuminating set of $K$ or, equivalently, that $F$ primitively illuminates $K$. Grünbaum [3] (see also p. 423 of [4]) suggested the question about the maximum number of points in a primitive illuminating set of a convex body $K \subset E^{d}$. This maximum is easily shown to be 4 for $d=2$ (see p. 423 of [4]). Although even a proof for the existence of the maximum for $d \geq 3$ was lacking, Grünbaum [3] conjectured that any primitive illuminating set of a convex body in $E^{d}, d \geq 3$, has at most $2^{d}$ points.

V. Soltan [9] confirmed this conjecture for the case $d=3$. More exactly, he proved that any primitive illuminating set of a convex body in $E^{3}$ has at most eight points, and only convex polytopes combinatorially equivalent to the 3 -cube have primitive illuminating sets of eight points (placed at their vertices).

In this paper we give a negative answer to Grünbaum's conjecture for all $d \geq 4$, by proving the following assertion.

Theorem 1. For any positive integers $m \geq 2$ and $d \geq 4$, there exists a convex body $K_{m} \subset E^{d}$ with a primitive illuminating set of at least $m$ points.

The respective bodies are obtained by a slight modification of cyclic polytopes in $E^{d}$. Namely, given any even number of points $z_{1}, \ldots, z_{2 r}$ on the moment curve in $E^{d}$, we extend the line segments $\left[z_{1}, z_{2}\right], \ldots,\left[z_{2 r-1} z_{2 r}\right]$ to very thin spindle-shaped bodies $V_{1,2}, \ldots, V_{2 r-1,2 r}$ such that the convex hull of $V_{1,2} \cup \cdots \cup V_{2 r-1,2 r}$ is a convex body primitively illuminated by $z_{1}, \ldots, z_{2 r}$ and the points $z_{2 i-1}, z_{2 i}$ illuminate each other for all $i=1, \ldots, r$ (for the technical details, see the proof of Theorem 1).

A more elaborated technique of this modification enables us to prove even the following theorem.

Theorem 2. For each $d \geq 4$, there is a convex body $K \subset E^{d}$ such that for any positive integer $m$ it has a primitive illuminating set of at least $m$ points.

In connection with this result, one could ask whether there exist convex bodies having infinite primitive illuminating sets. The following simple proposition shows that this is impossible.

Proposition 1. Any primitive illuminating set of a convex body $K \subset E^{d}$ is finite.

A more restrictive notion of inner illumination was introduced by Hadwiger [5]: a convex $d$-polytope $P \subset E^{d}$ is said to be illuminated by its vertices provided for any vertex $x$ of $P$ there is another vertex $z$ of $P$ such that ] $x, z$ [ $\subset$ int $P$. Hadwiger [5] asked whether a convex $d$-polytope in $E^{d}$ illuminated by its vertices has at least $2 d$ vertices. Mani [6] proved that for $d \leq 7$ the answer to Hadwiger's question is affirmative, while for higher dimensions he showed the existence of a convex $d$-polytope illuminated by its vertices and having about $d+2 \sqrt{d}$ vertices. 
Proposition 2. A convex d-polytope $P \subset E^{d}$ primitively illuminated by its vertices has at least $2 d$ vertices.

At the end of this section we pose the following problem.

Problem. Does there exist a least positive integer $k(d), d \geq 1$, such that any convex $d$-polytope in $E^{d}$ primitively illuminated by its vertices has at most $k(d)$ vertices?

Clearly, $k(1)=2, k(2)=4$, and from [9] it follows that $k(3)=8$.

\section{Proofs}

To prove Theorems 1 and 2 we need some auxiliary lemmas. Recall that a subset $Q$ of a convex body $K$ is called extreme provided for any points $x, z \in K$ the property ]$x, z[\cap Q \neq \emptyset$ implies $x, z \in Q$. Clearly, both the extreme subset $Q$ and its complement $K \backslash Q$ are convex sets. The following lemma is obvious.

Lemma 1. For any boundary point $x$ of a convex body $K \subset E^{d}$, the set

$$
Q_{x}:=\bigcup\{[v, w]: x \in] v, w[\subset K\}
$$

is the minimal (under inclusion) extreme subset of $K$ containing $x$ in its relative interior: $x \in \operatorname{rint} Q_{x}$.

Lemma 2. Let $K \subset E^{d}$ be a convex body and let $G$ be a subset of bd $K$ such that $K=\operatorname{conv} G$. A set $F \subset$ bd $K$ illuminates $K$ from within if and only if $F$ illuminates $G$, i.e., iffor any point $g \in G$ there is a point $f \in F$ satisfying $] f, g[\subset$ int $K$.

Proof. Since the "only if" part of the assertion is trivial, it remains to prove the "if" part. Let $G$ be a subset of bd $K$ such that $K=\operatorname{conv} G$, and let a set $F \subset$ bd $K$ illuminate $G$.

Choose an arbitrary point $x \in$ bd $K$. If $x \in G$, then $x$ is illuminated by a point from $F$. Assume that $x \notin G$, and denote by $Q_{x}$ the least under inclusion extreme subset of $K$ containing $x$ in its relative interior (see Lemma 1). The set $Q_{x}$ contains a point $g \in G$, since otherwise conv $G \subset K \backslash Q_{x}$, contrary to $K=\operatorname{conv} G$. Let $f \in F$ be a point illuminating $g$. Due to the construction of $Q_{x}$, there is a point $h \in Q_{x}$ such that $\left.x \in\right] g, h[$. From here and the inclusion $] f, g[\subset$ int $K$ it easily follows that $] f, x[\subset$ int $K$. Hence $F$ illuminates $K$ from within.

Two more lemmas deal with some properties of the moment curve $M \subset E^{d}$, defined parametrically by $x(t)=\left(t, t^{2}, \ldots, t^{d}\right), t \geq 0$. It is well known (see, e.g., [2] and p. 61 of [4]) that for a given positive integer $k$, with $2 k \leq d$, any $k$ points in $M$ determine a $(k-1)$-dimensional face of the convex set conv $M$. We need a particular case of this assertion, namely $k=1$. 
Lemma 3. Let $z^{\prime}=x(\eta)$ and $z^{\prime \prime}=x(\theta), 0<\eta \leq \theta$, be two points on the moment curve $M \subset E^{d}, d \geq 4$. Then the half-space $H\left(z^{\prime}, z^{\prime \prime}\right)$ defined by

$$
\left.\begin{array}{c}
H\left(z^{\prime}, z^{\prime \prime}\right)=\left\{v \in E^{d}:\langle v, b\rangle \leq \beta\right\}, \quad b=\left(b_{1}, \ldots, b_{d}\right), \\
b_{1}=-2 \eta \theta(\eta+\theta), \quad b_{2}=\eta^{2}+4 \eta \theta+\theta^{2}, \quad b_{3}=-2(\eta+\theta), \\
b_{4}=1, \quad b_{5}=\cdots=b_{d}=0, \quad \beta=-(\eta \theta)^{2},
\end{array}\right\}
$$

supports $M$ such that $H\left(z^{\prime}, z^{\prime \prime}\right) \cap M=\left\{z^{\prime}, z^{\prime \prime}\right\}$.

Proof. Indeed, for any point $z=\left(t, t^{2}, \ldots, t^{d}\right) \in M$ one has

$$
\langle z, b\rangle-\beta=b_{1} t+b_{2} t^{2}+b_{3} t^{3}+b_{4} t^{4}-\beta=(t-\eta)^{2}(t-\theta)^{2} .
$$

Since the last expression is nonnegative and equals 0 if and only if $t=\eta$ or $t=\theta$, we conclude that $M$ lies in the closed half-space $\left\{v \in E^{d}:\langle v, b\rangle \geq \beta\right\}$ complementary to $H\left(z^{\prime}, z^{\prime \prime}\right)$ such that $H\left(z^{\prime}, z^{\prime \prime}\right) \cap M=\left\{z^{\prime}, z^{\prime \prime}\right\}$.

Lemma 4. Let $z_{1}=x\left(t_{1}\right), z_{2}=x\left(t_{2}\right), z_{0}=x\left(t_{0}\right), 0<t_{1}<t_{2}<t_{0}$, be three points on the moment curve $M \subset E^{d}, d \geq 4$. There is a real number $\varepsilon>0$ such that the ball with radius $\varepsilon$ and center $z_{1}$ is disjoint to any half-space $H\left(z^{\prime}, z^{\prime \prime}\right)$ of the form (1), with $t_{2} \leq \eta \leq \theta \leq t_{0}$.

Proof. Clearly, the distance from $z_{1}$ to $H\left(z^{\prime}, z^{\prime \prime}\right)$ equals $\left(\left\langle z_{1}, b\right\rangle-\beta\right) /\|b\|$. One has

$$
\left\langle z_{1}, b\right\rangle-\beta=\left(t_{1}-\eta\right)^{2}\left(t_{1}-\theta\right)^{2} \geq\left(t_{1}-t_{2}\right)^{4}
$$

and

$$
\begin{aligned}
\|b\| & =\langle b, b\rangle^{1 / 2}=\left(4 \eta^{2} \theta^{2}(\eta+\theta)^{2}+\left(\eta^{2}+4 \eta \theta+\theta^{2}\right)^{2}+4(\eta+\theta)^{2}+1\right)^{1 / 2} \\
& \leq\left(16 t_{0}^{6}+36 t_{0}^{4}+16 t_{0}^{2}+1\right)^{1 / 2} .
\end{aligned}
$$

Hence

$$
\mu:=\frac{\left(t_{1}-t_{2}\right)^{4}}{\left(16 t_{0}^{6}+36 t_{0}^{4}+16 t_{0}^{2}+1\right)^{1 / 2}} \leq \frac{\left\langle z_{1}, b\right\rangle-\beta}{\|b\|},
$$

and any real number $\varepsilon \in] 0, \mu[$ satisfies the condition of Lemma 2.

In the proof of Theorems 1 and 2 we use the following notation. For any points $z_{i}, z_{j}$ on the moment curve $M, w_{i, j}$ denotes the middle point of the line segment $\left[z_{i}, z_{j}\right]$. If $\varepsilon_{i, j}$ is a real positive number, then $U_{i, j}$ means the closed ball with center $w_{i, j}$ and radius $\varepsilon_{i, j}$, and $C_{i, j}$ denotes the open convex cone with apex $z_{i}$ generated by int $U_{i, j}$ :

$$
C_{i, j}=\left\{(1-\lambda) z_{i}+\lambda v: v \in \operatorname{int} U_{i, j}, \lambda>0\right\} .
$$

Put

$$
V_{i, j}=\operatorname{conv}\left(U_{i, j} \cup\left[z_{i}, z_{j}\right]\right) .
$$

In what follows, $H\left(z^{\prime}, z^{\prime \prime}\right)$ stands for a half-space of the form (1). 
Proof of Theorem 1. Let $m$ be any positive integer and let $z_{1}=x\left(t_{1}\right), \ldots, z_{2 r}=x\left(t_{2 r}\right)$, $0<t_{1}<\cdots<t_{2 r}$, be any $2 r$ points on the moment curve $M \subset E^{d}$, with $2 r \geq m$. Due to Lemma 3 , the middle point $w_{p, q}$ of a line segment $\left[z_{p}, z_{q}\right], 1 \leq p<q \leq 2 r$, lies outside any half-space $H\left(z_{i}, z_{j}\right), 1 \leq i<j \leq 2 r,\{i, j\} \neq\{p, q\}$. Hence there is a real number $\varepsilon_{p, q}$ such that the closed ball $U_{p, q}$ with center $w_{p, q}$ and radius $\varepsilon_{p, q}$ is disjoint to $H\left(z_{i}, z_{j}\right)$ for all $i, j$ satisfying $1 \leq i<j \leq 2 r,\{i, j\} \neq\{p, q\}$.

We claim that the set $Z:=\left\{z_{1}, \ldots, z_{2 r}\right\}$ primitively illuminates the convex body

$$
K:=\operatorname{conv}\left(V_{1,2} \cup V_{3,4} \cup \cdots \cup V_{2 r-1,2 r}\right) .
$$

First we observe that any line segment $\left[z_{i}, z_{j}\right], 1 \leq i<j \leq 2 r$, with $\{i, j\}$ not in

$$
\Omega:=\{\{1,2\},\{3,4\}, \ldots,\{2 r-1,2 r\}\},
$$

is a one-dimensional face of $K$ (due to the choice of the numbers $\varepsilon_{p, q}$ ). This implies that two points $z_{m}, z_{n} \in Z$ illuminate each other if and only if $\{m, n\} \in \Omega$. Hence no proper subset of $Z$ illuminates $Z$ and, subsequently, no proper subset of $Z$ illuminates $K$ from within.

It remains to show that $Z$ illuminates the whole body $K$. Indeed, it is easily seen that any convex body $V_{i, i+1},\{i, i+1\} \in \Omega$, is illuminated from within by the set $\left\{z_{i}, z_{i+1}\right\}$. As a result, the set

$$
G:=\bigcup\left\{\mathrm{bd} K \cap V_{i, i+1}:\{i, i+1\} \in \Omega\right\}
$$

is illuminated by $Z$. Since $K=\operatorname{conv} G$, we get from Lemma 2 that $Z$ illuminates the whole body $K$.

Proof of Theorem 2. The required convex body $K \subset E^{d}$ will be represented as the union of an increasing sequence $K_{1} \subset \cdots \subset K_{r} \subset \cdots$ of convex bodies like those in the proof of Theorem 1 . The bodies $K_{1}, \ldots, K_{r}, \ldots$ are constructed recursively, by induction on $r$.

Choose two distinct points $z_{1}=x\left(t_{1}\right)$ and $z_{0}=x\left(t_{0}\right), 0<t_{1}<t_{0}$, on the moment curve $M \subset E^{d}$, and let $M_{0} \supset M_{1} \supset \cdots$ be a decreasing sequence of arcs of $M$ such that $M_{0}=\left\{x(t): t_{1} \leq t \leq t_{0}\right\}$ and $\bigcap\left\{M_{i}: i \geq 0\right\}=\left\{z_{0}\right\}$.

Initial step. The first body $K_{1}$ is determined by four points

$$
z_{1}=x\left(t_{1}\right), \quad z_{2}=x\left(t_{2}\right), \quad z_{3}=x\left(t_{3}\right), \quad z_{0}=x\left(t_{0}\right), \quad 0<t_{1}<t_{2}<t_{3}<t_{0},
$$

on the moment curve $M$. Namely, put

$$
K_{1}:=\operatorname{conv}\left(V_{1,0} \cup V_{2,3}\right),
$$

where $V_{1,0}$ and $V_{2,3}$ are as in (3) and real numbers $\varepsilon_{1,0}>0$ and $\varepsilon_{2,3}>0$ are defined as follows:

(a $\varepsilon_{1,0}$ is the radius of a ball $U_{1,0}$ disjoint to any of the half-spaces

$$
H\left(z_{1}, z_{2}\right), \quad H\left(z_{1}, z_{3}\right), \quad \text { and } \quad H(x(\eta), x(\theta)), \quad t_{2} \leq \eta \leq \theta \leq t_{0},
$$

and it is so small that the intersection $M \cap C_{1,0}$ is a subset of $M_{1}$ and contains none of $z_{2}, z_{3}$; 
( $\left.\mathrm{a}_{2}\right) \varepsilon_{2,3}$ is the radius of a ball $U_{2,3}$ disjoint to any of the half-spaces

$$
\begin{aligned}
& H\left(z_{i}, z_{j}\right), \quad i, j=0,1,2,3, \quad\{i, j\} \neq\{2,3\} \\
& \text { and } \quad H(x(\eta), x(\theta)), \quad t_{3} \leq \eta \leq \theta \leq t_{0}
\end{aligned}
$$

The existence of $\varepsilon_{1,0}$ and $\varepsilon_{2,3}$ follows from Lemmas 3 and 4 . Indeed, let $\varepsilon>0$ be a real number such that the ball with center $z_{1}$ and radius $\varepsilon$ is disjoint to any half-space $H(x(\eta), x(\theta)), t_{2} \leq \eta \leq \theta \leq t_{0}$. Since both $z_{1}$ and $z_{0}$ lie in all closed half-spaces complementary to these half-spaces, the middle point $w_{1,0}$ of the line segment $\left[z_{1}, z_{0}\right]$ is at a distance at least $\varepsilon / 2$ from any $H(x(\eta), x(\theta)), t_{2} \leq \eta \leq \theta \leq t_{0}$. Now, by Lemma 3, one can choose $\left.\varepsilon_{1,0} \in\right] 0, \varepsilon / 2$ [ such that the ball $U_{1,0}$ with center $w_{1,0}$ and radius $\varepsilon_{1,0}$ is disjoint to both half-spaces $H\left(z_{1}, z_{2}\right)$ and $H\left(z_{1}, z_{3}\right)$. The existence of $\varepsilon_{2,3}$ can be shown similarly.

It is easily seen (compare with the proof of Theorem 1 ) that the points $z_{0}, z_{1}, z_{2}, z_{3}$ primitively illuminate $K_{1}$, and $z_{p}$ illuminates $z_{q}, p, q=0,1,2,3$, if and only if $\{p, q\}=$ $\{0,1\}$ or $\{p, q\}=\{2,3\}$.

Inductive step. Assume that for a given positive integer $r(\geq 2)$ some $3 r-2$ points

$$
z_{1}=x\left(t_{1}\right), \quad \ldots, \quad z_{3 r-3}=x\left(t_{3 r-3}\right), \quad z_{0}=x\left(t_{0}\right) \in M, \quad t_{1}<\cdots<t_{3 r-3}<t_{0},
$$

and some real positive numbers $\varepsilon_{1,0}, \varepsilon_{2,3}, \varepsilon_{4,0}, \varepsilon_{5,6}, \ldots, \varepsilon_{3 r-5,0}, \varepsilon_{3 r-4,3 r-3}$ are chosen such that:

( $\left.\mathrm{b}_{1}\right) M \cap C_{3 i-2,0}$ is a subset of $M_{3 i-2}, i=1, \ldots, r-1$,

$\left(\mathrm{b}_{2}\right)$ the points $z_{1}, \ldots, z_{3 i}$ lie in the set $M_{0} \backslash C_{3 i-2,0}, i=1, \ldots, r-1$,

$\left(\mathrm{b}_{3}\right)$ the points $z_{3 i+1}, \ldots, z_{3 r-3}$ lie in the set $M \cap C_{3 i-2,0}, i=1, \ldots, r-2$,

( $\left.\mathrm{b}_{4}\right) \varepsilon_{3 i-2,0}$ is the radius of a ball $U_{3 i-2,0}, i=1, \ldots, r-1$, disjoint to any of the half-spaces

$$
H\left(z_{p}, z_{q}\right), \quad p, q=0,1, \ldots, 3 i, \quad\{p, q\} \neq\{0,3 i-2\},
$$

as well as to any of the half-spaces $H(x(\eta), x(\theta)), t_{3 i-1} \leq \eta \leq \theta \leq t_{0}$,

(b) $\varepsilon_{3 i-1,3 i}$ is the radius of a ball $U_{3 i-1,3 i}, i=1, \ldots, r-1$, disjoint to any of the half-spaces

$$
H\left(z_{p}, z_{q}\right), \quad p, q=0,1, \ldots, 3 i, \quad\{p, q\} \neq\{3 i-1,3 i\},
$$

as well as to any of the half-spaces $H(x(\eta), x(\theta)), t_{3 i} \leq \eta \leq \theta \leq t_{0}$.

Now we choose three new points

$$
z_{3 r-2}=x\left(t_{3 r-2}\right), \quad z_{3 r-1}=x\left(t_{3 r-1}\right), \quad z_{3 r}=x\left(t_{3 r}\right), \quad t_{3 r-3}<t_{3 r-2}<t_{3 r-1}<t_{3 r}<t_{0},
$$

and real positive numbers $\varepsilon_{3 r-2,0}, \varepsilon_{3 r-1,3 r}$ such that:

(c $\left.c_{1}\right) M \cap C_{3 r-2,0}$ is a subset of $M_{3 r-2}$,

(c $\left.c_{2}\right)$ the points $z_{1}, \ldots, z_{3 r}$ lie in the set $M_{0} \backslash C_{3 r-2,0}$,

$\left(c_{3}\right)$ the points $z_{3 r-2}, z_{3 r-1}, z_{3 r}$ lie in the set $M \cap C_{3 r-2,0}$, 
(c $\varepsilon_{4} \varepsilon_{3 r-2,0}$ is the radius of a ball $U_{3 r-2,0}$ disjoint to any of the half-spaces

$$
H\left(z_{p}, z_{q}\right), \quad p, q=0,1, \ldots, 3 r, \quad\{p, q\} \neq\{0,3 r-2\},
$$

as well as to any of the half-spaces $H(x(\eta), x(\theta)), t_{3 r-1} \leq \eta \leq \theta \leq t_{0}$,

(c5) $\varepsilon_{3 r-1,3 r}$ is the radius of a ball $U_{3 r-1,3 r}$ disjoint to any of the half-spaces

$$
H\left(z_{p}, z_{q}\right), \quad p, q=0,1, \ldots, 3 r, \quad\{p, q\} \neq\{3 r-1,3 r\},
$$

as well as to any of the half-spaces $H(x(\eta), x(\theta)), t_{3 r} \leq \eta \leq \theta \leq t_{0}$.

As a result (compare with the proof of Theorem 1), the convex body

$$
K_{r}:=\operatorname{conv}\left(V_{1,0} \cup V_{2,3} \cup V_{4,0} \cup V_{5,6} \cup \cdots \cup V_{3 r-2,0} \cup V_{3 r-1,3 r}\right)
$$

is primitively illuminated by any of the sets

$$
Z_{i}:=\left\{z_{0}, z_{3 i-2}\right\} \cup\left\{z_{2}, z_{3} ; z_{5}, z_{6} ; \ldots ; z_{3 i-1}, z_{3 i}\right\}, \quad i=1, \ldots, r,
$$

such that two points $z_{p}, z_{q} \in Z_{i}$ illuminate each other if and only if $\{p, q\}$ is one of the pairs $\{0,3 i-2\},\{2,3\},\{5,6\}, \ldots,\{3 i-1,3 i\}$.

Now put $K=K_{1} \cup K_{2} \cup \cdots$. From the construction above it follows that $K$ is a convex body illuminated from within by the set $Z:=\left\{z_{0}, z_{1}, \ldots\right\}$ such that:

$\left(\mathrm{d}_{1}\right)$ any point $z_{3 m-2}, m \geq 1$, illuminates $z_{0}$ and all points $z_{n}, n \geq 3 m+1$,

$\left(\mathrm{d}_{2}\right)$ for any $m \geq 1$ the points $z_{3 m-1}$ and $z_{3 m}$ illuminate each other,

$\left(\mathrm{d}_{3}\right)$ for any pair of points $z_{p}, z_{q} \in Z$ not mentioned in $\left(\mathrm{d}_{1}\right)$ and $\left(\mathrm{d}_{2}\right)$, i.e., such that

$$
\{p, q\} \notin\{\{0,3 m-2\},\{3 m-2, n\}, m \geq 1, n \geq 3 m+1\} \cup\{\{3 m-1,3 m\}, m \geq 1\},
$$

the line segment $\left[z_{p}, z_{q}\right]$ is in the boundary of $K$.

Hence for any positive integer $m \geq 1$ the set

$$
\left\{z_{0}, z_{3 m-2}\right\} \cup\left\{z_{2}, z_{3} ; z_{5}, z_{6} ; \ldots ; z_{3 m-1}, z_{3 m}\right\}
$$

of cardinality $2 m+2$ primitively illuminates $K$.

Proof of Proposition 1. It is sufficient to show that any set $F$ illuminating a convex body $K$ from within has a finite subset also illuminating $K$ from within. To do this, consider for any point $x \in F$ the set $I(x)$ of all points in bd $K$ illuminated by $x$ :

$$
I(x):=\{z \in \text { bd } K:] x, z[\subset \operatorname{int} K\} .
$$

We claim that $I(x)$ is a relatively open set in the boundary of $K$. Indeed, let $v$ be any point in $I(x)$, and denote by $w$ the middle point of the open line segment $] x, v[$. Since $w \in$ int $K$, there is an open ball $U \subset K$ with center $w$. Clearly, the set $N(v)=\{z \in$ bd $K:] x, z[\cap U\}$ is a relatively open neighborhood of $v$ in bd $K$.

The fact that $F$ illuminates $K$ from within means that the family of sets $\{I(x): x \in F\}$ covers bd $K$. By the compactness argument, one can choose a finite subset $F^{\prime}$ of $F$ such that bd $K=\bigcup\left\{I(x): x \in F^{\prime}\right\}$. In other words, the set $F^{\prime}$ illuminates $K$ from within. 
Proof of Proposition 2. It is shown in [6] (see also [7]) that if a convex $d$-polytope $P \subset E^{d}$ is illuminated by its vertex set $V(P)$ in such a way that any vertex of $P$ illuminates exactly one other vertex, then $P$ has at least $2 d$ vertices. Hence it is sufficient to prove that if $P$ is primitively illuminated by $V(P)$, then any vertex of $P$ illuminates exactly one other vertex.

Indeed, assume for contradiction that there are three vertices $v_{1}, v_{2}, v_{3} \in V(P)$ such that $v_{2}$ illuminates both $v_{1}$ and $v_{3}$. Since the set $V(P)$ primitively illuminates $P$ and $\left\{v_{1}, v_{2}\right\}$ illuminates $\left\{v_{1}, v_{2}, v_{3}\right\}$, there is a new vertex $v_{4} \in V(P) \backslash\left\{v_{1}, v_{2}, v_{3}\right\}$ illuminated by $v_{3}$ and not illuminated by any of $v_{1}, v_{2}$. By a similar argument, for any positive integer $r \geq 3$ there is a vertex $v_{r+1} \in V(P) \backslash\left\{v_{1}, \ldots, v_{r}\right\}$ illuminated by $v_{r}$ and not illuminated by any of $v_{1}, \ldots, v_{r-1}$. The last is in contradiction to the finiteness of $V(P)$.

\section{References}

1. V. Boltyanski, H. Martini, and P. Soltan, Excursions into Combinatorial Geometry, Springer-Verlag, Berlin, 1997.

2. D. Gale, Neighborly and cyclic polytopes, Proc. Symp. Pure Math. Vol. VII. Convexity (Seattle, 1961), pp. 225-232. American Mathematical Society, Providence, RI, 1963.

3. B. Grünbaum, Fixing systems and inner illumination, Acta Math. Acad. Sci. Hungar. 15 (1964), 161-163.

4. B. Grünbaum, Convex Polytopes, Interscience, London, 1967.

5. H. Hadwiger, Ungelöstes Problem Nr. 55, Elem. Math. 27 (1972), 57.

6. P. Mani, Inner illumination of convex polytopes, Comment. Math. Helv. 49 (1974), 65-73.

7. M. Rosenfeld, Inner illumination of convex polytopes, Elem. Math. 30 (1975), 27-28.

8. P. Soltan, On the illumination of the boundary of a convex body from within (Russian), Mat. Sb. 57 (1962), 443-448.

9. V. Soltan, On Grünbaum's problem about inner illumination of convex bodies, Acta Math. Hungar. 69 (1995), 15-25.

Received December 1, 1997, and in revised form January 21, 1999. 